\title{
La circulación de libros en la Biblioteca de la Universidad de León (España)
}

\author{
Blanca Rodríguez-Bravo* \\ Francisco Jesus Rodríguez-Sedano**
}

Artículo recibido:

21 de septiembre de 2018

Artículo aceptado:

10 de enero de 2019

Artículo de investigación

\section{Resumen}

El objetivo general de esta investigación se dirige a conocer la circulación de los materiales tradicionales en las bibliotecas de la ULe, para observar la evolución del préstamo en el momento de auge de la colección electrónica, indagar sobre los principales usuarios de la colección y averiguar qué tipos documentales son los más prestados. En especial interesa saber el posicionamiento del libro como fuente de información en la comunidad universitaria. De los resultados obtenidos se desprende que el volumen de la colección se adecua al número de

* Departamento de Biblioteconomía y Documentación, Universidad de León, España blanca.rodriguez@unileon.es

** Departamento de Ingeniería Eléctrica, Informática y Aeronáutica, Universidad de León, España 
usuario, que el movimiento de la colección no es muy elevado, aunque existen notables diferencias entre centros, y que el préstamo, al igual que la colección, está dominado por los libros impresos.

Palabras clave: Bibliotecas Universitarias; Circulación de Colecciones; Libros; Préstamo; Universidad de León.

\section{Books circulation at the Library of the University of Leon (Spain) \\ Blanca Rodríguez-Bravo and Francisco Jesus Rodríguez- Sedano}

\section{Abstract}

The aim of this research was to determine the circulation of materials held in the University of León's (ULe) library in order to identify: loan trends; the main users of the collection, and the types of document most frequently loaned. The major interest was to find out the relevance of the book as an information resource. We found that the size of the collection in relation to the number of users was adequate, that the circulation of the collection was relatively low, although with notable differences between libraries, and that loans, as well as the collection are dominated by printed books.

Keywords: Books; Collection Circulation; Loans University Libraries; University of León (Spain).

\section{INTRODUCCIÓN}

T os estudios de circulación de colecciones de monografías en bibliotecas Luniversitarias realizados durante la última década ponen de manifiesto que, a medida que las bibliotecas universitarias incrementan su inversión en la adquisición de recursos electrónicos, se reduce la colección impresa y también su circulación (Martell, 2008; Rose-Wiles, 2011).

Así, el informe de la Association of Research Libraries (ARL) 2008-2009 ya indicaba que las bibliotecas gastaban más del 50 por ciento de su presupuesto 
en la adquisición de documentos en materiales electrónicos. Respecto de la colección tradicional, constataba también que, si bien el presupuesto dedicado a revistas entre 1991 y 2009 se había incrementado ligeramente, la proporción invertida en monografías se había reducido (Kyrillidou y Morris, 2011).

El trabajo de Simón-Martín et al. (2016), cuyo objetivo fue examinar el impacto de la crisis financiera en las bibliotecas de las universidades públicas españolas durante el periodo 2008-2014, afirma igualmente que estas bibliotecas han experimentado una reducción en los gastos de adquisiciones de recursos de información y que el gasto en recursos electrónicos se ha incrementado, a expensas del gasto en monografías y en revistas en soporte papel. Esta situación se confirma con los datos de la evolución del gasto en la ULe que mostramos párrafos adelante.

Sin embargo, creemos que los libros son todavía de uso prioritario para la docencia, sin perjuicio de su utilización para fines de investigación. Se trata de las fuentes de mayor uso de los estudiantes, grupo más numeroso en la comunidad académica universitaria. Esta situación se ha constatado en un estudio previo (Rodríguez-Bravo et al., 2015) en el que se analizaron las bibliografías de las asignaturas de once titulaciones de tres universidades, incluida la de León, a través de sus guías docentes del curso 2013-2014, y se observó que los libros son los recursos dominantes. El porcentaje de documentos electrónicos en las bibliografías de las guías docentes es, por el contrario, todavía muy escaso. En las tres universidades se mantiene por debajo de un 5 por ciento del total.

Otro estudio previo analizó también el ciclo anual del préstamo de documentos en la ULe (Rodríguez-Bravo y Rodríguez-Sedano, 2016).

\section{Objetivos y metodología}

El objetivo general de esta investigación se dirige a conocer la circulación de los libros (ya sean monografías o manuales) en las bibliotecas de la ULe, para observar la evolución del préstamo en el momento de auge de la colección electrónica, indagar sobre los principales usuarios de la colección y averiguar qué tipos documentales son los más prestados. Consideramos que los resultados de este estudio servirán de guía para establecer una política de desarrollo de las colecciones y que serán representativos de lo que acaece en otras bibliotecas universitarias españolas.

La Universidad de León es una universidad pública de tamaño medio en el entorno español situada en el noroeste de este país. 


\begin{tabular}{|l|r|r|r|r|r|}
\hline & $2011-2012$ & $2012-2013$ & $2013-2014$ & $2014-2015$ & $2015-2016$ \\
\hline Alumnos & 13,755 & 13,106 & 12,895 & 12,515 & 10,925 \\
\hline $\begin{array}{l}\text { Personal docente } \\
\text { e investigador }\end{array}$ & 936 & 930 & 854 & 854 & 909 \\
\hline $\begin{array}{l}\text { Personal de } \\
\text { administración } \\
\text { y servicios }\end{array}$ & 543 & 526 & 514 & 499 & 503 \\
\hline \multicolumn{1}{|c|}{ Totales } & 15,234 & 14,562 & 14,263 & 13,868 & 12,337 \\
\hline
\end{tabular}

Cuadro 1. Cifras de la comunidad universitaria de la ULe/curso. Fuente: excepto donde se señale, todos los elementos gráficos son elaboración nuestra.

Los datos del Cuadro 1 muestran una disminución lenta en las cifras de la comunidad universitaria hasta el curso 2015-2016, cuando hubo una caída mayor, debido a la disminución acusada del alumnado de nuevo ingreso, que no ha podido compensar el ligero aumento durante dicho curso del profesorado y del personal de administración y servicios respecto del curso 2014-2015.

Como se observa en el Cuadro 2, las áreas de Ciencias Sociales y Ciencias Técnicas tienen mayor número de estudiantes. No obstante, son las áreas de Ciencias de la Naturaleza y de la Salud las que más profesorado reúnen y las más activas en el ámbito de la investigación:

\begin{tabular}{|l|r|r|r|r|r|}
\hline & $2011-2012$ & $2012-2013$ & $2013-2014$ & $2014-2015$ & $2015-2016$ \\
\hline $\begin{array}{l}\text { Arte y } \\
\text { Humanidades }\end{array}$ & 936 & 917 & 858 & 878 & 838 \\
\hline $\begin{array}{l}\text { Ciencias de la } \\
\text { Salud }\end{array}$ & 2,075 & 1,559 & 1,857 & 1,623 & 1,591 \\
\hline Ciencias & 1,403 & 1,372 & 1,203 & 1,152 & 1,154 \\
\hline $\begin{array}{l}\text { Ingenieríay } \\
\text { Arquitectura }\end{array}$ & 3,613 & 3,902 & 3,938 & 4,149 & 2,836 \\
\hline $\begin{array}{l}\text { Sociales y } \\
\text { Jurídicas (excepto } \\
\text { educación) }\end{array}$ & 4,421 & 4,727 & 4,090 & 3,824 & 3,467 \\
\hline $\begin{array}{l}\text { Sociales y } \\
\text { Jurídicas (solo } \\
\text { educación) }\end{array}$ & 1,307 & 629 & 949 & 889 & 1,039 \\
\hline Total & 13,755 & 13,106 & 12,895 & 12,515 & 10,925 \\
\hline
\end{tabular}

Cuadro 2. Alumnos de la ULe por ramas de conocimiento/curso. 
Los objetivos específicos son los siguientes:

- Conocer la evolución de los datos de circulación.

- Indagar sobre quiénes son los principales usuarios de la colección de recursos disponible en préstamo.

- Averiguar qué tipos documentales son los más prestados.

Para realizar este estudio, se obtuvieron los ficheros de circulación que proporciona el Sistema Integrado de Gestión Bibliotecaria (SIGB) Innopac Millenium, desde el curso 2011-2012 hasta el 2015-2016. Concretamente ficheros mensuales de préstamos.

Por tipos de usuarios de los que hemos tomado en consideración a los tres grupos principales que componen la comunidad universitaria: estudiantes, personal docente e investigador (PDI) y personal de administración y servicios (PAS):

- Por tipos de materiales que distinguen: manuales y bibliografía recomendada, monografías, tesis y proyectos/trabajos de fin de carrera/ grado o máster, publicaciones periódicas, materiales especiales $(\mathrm{cd}$, audio, video, etc.), obras de referencia, material cartográfico y fondo antiguo.

- Por bibliotecas/áreas científicas.

El volumen de préstamos se ha puesto en relación con las cifras de miembros de la comunidad académica durante los cursos analizados obtenidos de los "Datos básicos del sistema universitario de Castilla y León", proporcionados por la Junta de Castilla y León (2018).

Asimismo, los datos de préstamo se han relacionado con las cifras globales de la colección disponible o accesible para realizar una primera aproximación al volumen relativo de la circulación en la biblioteca de la ULe. Para este fin, se han utilizado los datos que la biblioteca de esta universidad ha proporcionado a los anuarios de Rebiun ${ }^{1}$ (la Red de Bibliotecas Universitarias Española).

Igualmente, se comparan los datos obtenidos con los extraídos del análisis de la bibliografía recomendada en las guías docentes de las asignaturas, las cuales conforman las titulaciones impartidas en la ULe (Rodríguez-Bravo et al., 2015). 
La colección de la ULe

Partiremos en nuestro análisis de los datos de la colección de la biblioteca proporcionados por Rebiun. El total de recursos de la Biblioteca Universitaria de la ULe se desglosa de la forma siguiente (Cuadro 3):

\begin{tabular}{|l|r|r|r|r|r|}
\hline & \multicolumn{1}{|c|}{2011} & \multicolumn{1}{c|}{2012} & \multicolumn{1}{c|}{2013} & \multicolumn{1}{c|}{2014} & \multicolumn{1}{c|}{2015} \\
\hline Títulos de monografías en papel & 315,949 & 484,093 & 332,210 & 338,551 & 344,275 \\
\hline $\begin{array}{l}\text { Ítems de monografías en papel } \\
\text { informatizadas }\end{array}$ & 472,950 & 472,531 & 486,947 & 494,894 & 501,929 \\
\hline $\begin{array}{l}\text { Títulos de monografías audiovi- } \\
\text { suales y material no librario }\end{array}$ & - & 9,953 & - & - & 10,635 \\
\hline $\begin{array}{l}\text { Títulos de publicaciones periódi- } \\
\text { cas en papel en curso }\end{array}$ & 3,533 & 3,343 & 2,876 & 2,939 & 2,746 \\
\hline $\begin{array}{l}\text { Títulos de publicaciones periódi- } \\
\text { cas en papel muertas }\end{array}$ & 7,465 & 7,824 & 7,885 & 7,914 & 8,068 \\
\hline Documentos anteriores a 1900 & - & 3,629 & 3,637 & 1,783 & 3,796 \\
\hline $\begin{array}{l}\text { Monografías electrónicas de pago } \\
\text { o con licencia }\end{array}$ & 10,268 & 10,280 & 8,000 & 10,494 & 11,484 \\
\hline $\begin{array}{l}\text { Publicaciones periódicas de pago } \\
\text { o con licencia }\end{array}$ & 20,228 & 19,840 & 12,814 & 12,050 & 17,904 \\
\hline Recursos electrónicos propios & 1,040 & 1,940 & 2,125 & 6,614 & 48,053 \\
\hline
\end{tabular}

Cuadro 3. La colección de la Biblioteca de la ULe por tipos documentales/año.

Los datos cuantitativos de la colección disponible en la ULe, y que esta misma institución ha proporcionado a Rebiun, permiten observar una considerable estabilidad a lo largo de los cinco años estudiados. La excepción la constituyen los recursos electrónicos propios que han experimentado un crecimiento exponencial en 2015. Este crecimiento se advierte, asimismo, en las publicaciones periódicas suscritas a través de proveedores de contenidos que, tras descender significativamente en 2013 y 2014, se han vuelto a recuperar en 2015, aunque no alcanzan las cifras previas de 2011 ni de 2012.

La colección está, sin embargo, todavía conformada principalmente por libros impresos. Considerando el dato de títulos de monografías, no el de ítems, observamos que las monografías constituyen el 89 por ciento de la colección. 
En cuanto a la colección de e-books, ésta parece haber sufrido mayores altibajos con una disminución notable de las monografías electrónicas en 2013 y una recuperación posterior de las cifras previas en 2014, e incremento claramente visible en 2015. En cuanto a las publicaciones periódicas en papel en curso, han experimentado un progresivo descenso que guarda relación directa con el incremento de los títulos de publicaciones en papel cerrados. El material no librario muestra una tendencia a la alza.

La falta de crecimiento de la colección obedece al descenso presupuestario experimentado en los últimos cinco años en la biblioteca universitaria, reflejo de esa realidad en la universidad en su conjunto. Mostramos a continuación la evolución del gasto en euros en la colección de la ULe (Cuadro 4).

\begin{tabular}{|l|r|r|r|r|r|}
\hline & \multicolumn{1}{|c|}{2011} & \multicolumn{1}{c|}{2012} & \multicolumn{1}{c|}{2013} & \multicolumn{1}{c|}{2014} & \multicolumn{1}{c|}{2015} \\
\hline $\begin{array}{l}\text { Gasto en monografías en } \\
\text { papel }\end{array}$ & $481,890 €$ & $179,109 €$ & $164,664 €$ & $146,088 €$ & $176,847 €$ \\
\hline $\begin{array}{l}\text { Gasto en publicaciones } \\
\text { periódicas en papel }\end{array}$ & $259,480 €$ & $175,967 €$ & $130,395 €$ & $112,744 €$ & $107,492 €$ \\
\hline Gastos en e-books & $20,298 €$ & $13,669 €$ & $3,360 €$ & $58,779 €$ & $40,036 €$ \\
\hline Gasto en e-journals & $212,422 €$ & $238,739 €$ & $201,227 €$ & $190,707 €$ & $183,008 €$ \\
\hline
\end{tabular}

Cuadro 4. El gasto en libros y revistas en la colección de la ULe/año.

Resulta reseñable el grave descenso en la inversión en la colección de monografías en papel, que en 2012 se redujo a una tercera parte respecto de 2011, para continuar descendiendo hasta 2015, cuando la inversión ha repuntado tímidamente. No es ése el caso de la inversión en publicaciones periódicas en papel en las que el gasto ha seguido una tendencia descendente, lo que se ha reflejado en el aumento de publicaciones cerradas, como se observa en el Cuadro 3.

También el gasto en revistas electrónicas ha experimentado un descenso desde 2012, mientras que la tímida inversión en libros electrónicos parece irse consolidando, si bien se halla a bastante distancia respecto de la de $e$-journals.

El estudio de Simón-Martín et al. (2016) señala que las bibliotecas universitarias españolas han experimentado una reducción en los gastos de adquisiciones de recursos de información. En el conjunto de la nación, la adquisición de monografías se redujo en un 47.0 por ciento y la de publicaciones periódicas en papel en un 52.8 por ciento. Asimismo, constata que el gasto en recursos electrónicos se ha incrementado entre 2008 y 2014, a expensas del gasto en monografías y en revistas impresas. 


\section{Datos globales de préstamos por tipo de usuario y curso académico}

Los datos absolutos de los préstamos que se presentan en el Cuadro 5 permiten observar una disminución en el número de préstamos en los tres estamentos que componen la comunidad universitaria. Si consideramos los datos globales de los cursos 2011-2012 y 2015-2016, la caída del número de transacciones de préstamos asciende al 29 por ciento. El Cuadro 5 muestra, asimismo, datos relativos que permiten observar la media de número de préstamos por usuario:

\begin{tabular}{|l|r|r|r|r|r|r|r|r|r|r|}
\hline & \multicolumn{2}{|c|}{$2011-2012$} & \multicolumn{2}{|c|}{$2012-2013$} & $2013-2014$ & \multicolumn{2}{|c|}{$2014-2015$} & \multicolumn{2}{|c|}{ 2015-2016 } \\
\hline & Número & Media & Número & Media & Número & Media & Número & Media & Número & Media \\
\hline Estudiantes & 110,226 & 8.01 & 98,352 & 7.50 & 96,037 & 7.45 & 84,791 & 6.78 & 73,739 & 6.75 \\
\hline Profesores & 11,178 & 11.94 & 8,656 & 9.31 & 4.546 & 5.32 & 9,644 & 11.29 & 7,946 & 8.74 \\
\hline $\begin{array}{l}\text { Personal } \\
\text { Admin/Serv. }\end{array}$ & 594 & 1.09 & 1,780 & 3.38 & 394 & 0.77 & 646 & 1.29 & 1,245 & 2.48 \\
\hline Otros usuarios & 613 & & 2,353 & & 1,636 & & 4,134 & & 4,144 & \\
\hline TOTAL & 122,611 & & 111,141 & & & & 99,215 & & 87,074 & \\
\hline
\end{tabular}

Cuadro 5. Préstamos totales por tipos de usuario/curso.

Como resulta obvio, el movimiento principal de la colección es efectuado por los estudiantes, al menos en términos globales, porque, en términos relativos, como era esperable, el uso más activo de la colección proviene del personal docente e investigador.

Las ratios de circulación por estudiante son reducidas. Los estudiantes no utilizan la bibliografía recomendada de manera muy activa, pese a que el espacio europeo de educación superior promueve la evaluación continua y con ésta la realización de lecturas, elaboración de trabajos, etc. Cabe señalar que el uso que realiza el personal de administración y servicios del servicio de préstamo durante los dos primeros cursos analizados ha sido significativamente elevado.

A nivel nacional, el trabajo de Simón-Martín et al. (2016) constata también la disminución de las transacciones de préstamo entre 2011 y 2014, que achacan a la disminución de los alumnos matriculados en las universidades públicas españolas durante ese periodo. Por lo que a la ULe se refiere, se advierte un descenso, ya que de algo más de ocho préstamos por curso y alumno en el curso 2011-2012, desciende a menos de siete préstamos en el curso 2015-2016. Este descenso se observa, igualmente, en los restantes colectivos donde se constata el descenso del uso del servicio durante los últimos cursos. 
Consideramos que el descenso de préstamos no resulta explicado totalmente por la disminución de los miembros de la comunidad académica. Pensamos que esta circunstancia puede estar correlacionada, asimismo, con un cambio de hábitos y una tendencia a utilizar complementariamente la colección electrónica, y también con la posibilidad de que la colección en papel no se esté renovando al ritmo que sería necesario, para adecuarse a las necesidades de los usuarios de la universidad, habida cuenta de las restricciones presupuestarias de los últimos años. En los Cuadros 3 y 4 se constata que la ULe ha puesto un énfasis especial en la inversión en la colección digital.

\section{Datos globales de préstamos totales por tipo de material y por curso académico}

Las cifras de préstamo por tipos de materiales posibilitan conocer qué tipos documentales se constituyen en recursos esenciales para el aprendizaje, la docencia y la investigación (Cuadro 6).

\begin{tabular}{|l|r|r|r|r|r|}
\hline & $2011 / 2012$ & $2012 / 2013$ & $2013 / 2014$ & $2014 / 2015$ & 2015/2016 \\
\hline $\begin{array}{l}\text { Manuales y bibliografía } \\
\text { rec. }\end{array}$ & 35,185 & 34,904 & 33,447 & 30,650 & 28,376 \\
\hline Monografías & 15,880 & 16,154 & 16,573 & 16,887 & 15,367 \\
\hline $\begin{array}{l}\text { Material especial } \\
\text { (cd, audio, video) }\end{array}$ & 398 & 314 & 733 & 526 & 302 \\
\hline $\begin{array}{l}\text { Tesis, proyectos fin } \\
\text { de grado }\end{array}$ & 5,181 & 4,573 & 3,499 & 2,760 & 1,910 \\
\hline Referencia & 627 & 586 & 554 & 371 & 367 \\
\hline Material cartográfico & 82 & 45 & 31 & 39 & 40 \\
\hline Publicaciones periódicas & 3,401 & 2,851 & 2,880 & 2,578 & 2,54 \\
\hline Colecciones especiales & 30 & 27 & 21 & 31 & 18 \\
\hline
\end{tabular}

Cuadro 6. Préstamos por tipos de documentos/curso.

Las cifras de préstamos de los distintos materiales reflejan una disminución clara si nos fijamos en los datos de préstamos de los cursos 2011/2012 y de 2015/2016; no obstante, el descenso no ha sido lineal en todos los casos, observándose ligeros vaivenes en el caso de las publicaciones seriadas, los materiales especiales y los cartográficos.

Atendiendo a los préstamos diferenciados por tipo de material, cabe señalar el predominio absoluto de los manuales y las monografías. Hay que tener en cuenta, no obstante, que algunos de los documentos que se registran en las 
restantes categorías no han estado sujetos tradicionalmente a préstamo, limitándose su utilización a la sala de lectura, es el caso de las tesis y los proyectos de fin de carrera, las obras de referencia, el material cartográfico, el fondo antiguo y las publicaciones periódicas.

La bibliografía básica y complementaria que conforma las guías docentes o fichas de las diversas asignaturas y que se encuentran disponibles online a través de la página de la universidad muestran que los profesores confían y recomiendan a sus estudiantes mayoritariamente la consulta de manuales, monografías y obras de referencia, principalmente en formato papel, con una presencia limitada de recursos electrónicos y residual de otros documentos como son artículos, capítulos de libros y ponencias (Rodríguez-Bravo et al., 2015).

De hecho, se ha constatado que el libro constituye más de un 90 por ciento del total de referencias en todas y cada una de las titulaciones que muestran una considerable homogeneidad en la selección de recursos. Ello no es óbice para que se recomiende y consulte bibliografía más variada que los profesores facilitan durante el curso en el desarrollo del leccionario.

\section{Datos globales de préstamos totales de libros por usuario y centro}

Se muestra enseguida la evolución en los cursos estudiados de la circulación realizada por los principales usuarios, aquellos directamente involucrados en el proceso de enseñanza-aprendizaje, esto es, estudiantes y profesores, y el uso que hacen de las dos principales fuentes de información que se utilizan en la docencia y el estudio: los manuales y la bibliografía recomendada, y las monografías.

En líneas generales, se observa un uso de manuales y bibliografía recomendada muy elevado entre los estudiantes; mientras que entre el personal docente e investigador las monografías son los documentos más frecuentemente llevados en préstamo (Gráficas 1 y 2). 


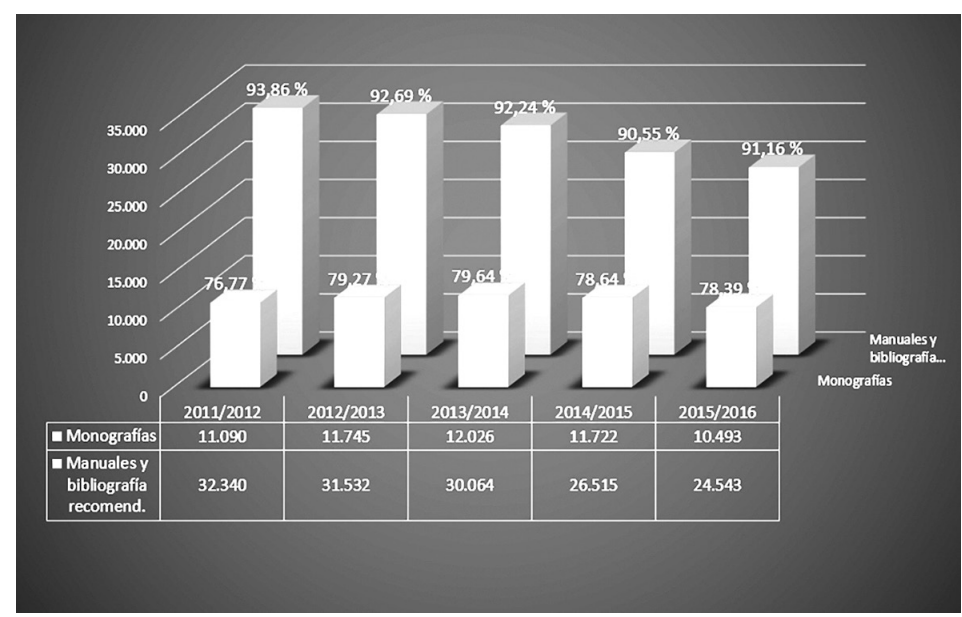

Gráfica 1. Préstamos libros/estudiantes.

En el caso de los estudiantes (Gráfica 1), es evidente la utilización preferente que realizan de los manuales y de la bibliografía recomendada en las guías docentes por los profesores. Son el principal recurso utilizado por los estudiantes para completar apuntes y resolver dudas. En el movimiento de manuales, se aprecia una considerable disminución, acorde con la de las cifras de estudiantes y con un uso complementario de e-books. Como señalan Simón-Martín y colegas (2016), el volumen del préstamo domiciliario en España ha seguido una evolución similar al del número de estudiantes matriculados.

Esta disminución se ha dado también en los dos últimos cursos, en la circulación de las monografías, que se constituye en el segundo tipo documental, pero con una circulación muy inferior, casi una tercera parte de las cifras de movimiento de los manuales.

En cuanto a los demás tipos documentales, los estudiantes también hacen un uso considerable de las tesis, además de otros trabajos finales y de las publicaciones periódicas (Anexo 1). 


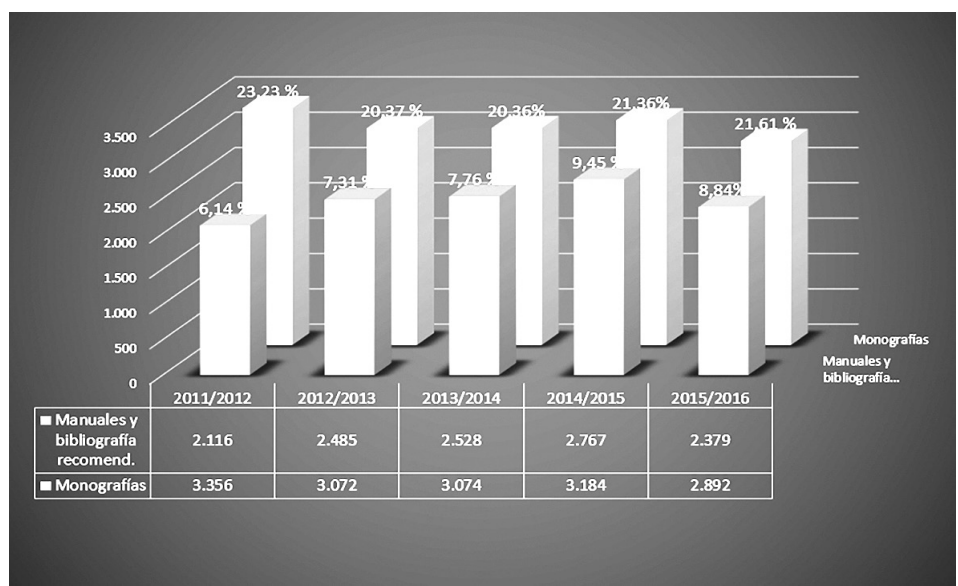

Gráfica 2. Préstamos libros/profesores.

En el caso de los profesores (Gráfica 2), se observa que la preferencia se invierte. Los profesores recurren más a las monografías que a los manuales, pero la distancia en el número de movimientos es inferior entre un tipo de material y otro, que en el caso de los estudiantes. El volumen de préstamos ha tenido altibajos y las cifras del último curso no son muy halagüeñas, pese al ligero incremento experimentado por el personal docente e investigador en el curso 2015-2016.

Al igual que en el caso de los estudiantes, esta situación obedecería a un superior uso de la colección electrónica que, por lo que a e-books se refiere, se ha consolidado por encima de los diez mil títulos, gracias a que la inversión presupuestaria en estos se ha incrementado desde 2014. 


\begin{tabular}{|c|c|c|c|c|c|c|c|c|c|c|c|c|c|c|}
\hline \multirow{4}{*}{ 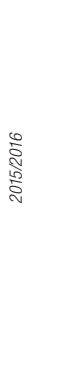 } & 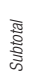 & 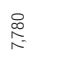 & 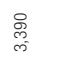 & $\begin{array}{l}\stackrel{0}{\infty} \\
\underset{\sim}{\sim}\end{array}$ & $\begin{array}{l}\text { o } \\
\text { ơ } \\
\text { c. }\end{array}$ & §ి & \begin{tabular}{|l|l}
0 \\
0 \\
0 \\
$\infty$ \\
$\infty$
\end{tabular} & 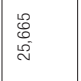 & 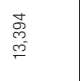 & \begin{tabular}{|l}
$\infty$ \\
o \\
i
\end{tabular} & \begin{tabular}{|l} 
\\
号 \\
尔
\end{tabular} & $\begin{array}{l}\text { 胥 } \\
\text { o }\end{array}$ & 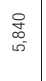 & $\begin{array}{l}\text { 売 } \\
\text { 总 }\end{array}$ \\
\hline & 题 & $\stackrel{8}{i}$ & 哭 & $\stackrel{q}{q}$ & ลี & \pm & 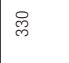 & 夺 & 悉 & $R$ & 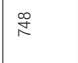 & $\dddot{8}$ & $\stackrel{8}{\circ}$ & 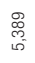 \\
\hline & 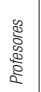 & 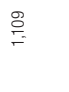 & 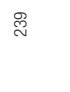 & $\approx$ & $\tilde{y}$ & I & 导 & 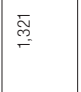 & 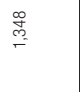 & Б్ & 兽 & $\stackrel{\circ}{\circ}$ & $\widehat{\widetilde{N}}$ & 景 \\
\hline & 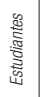 & $\begin{array}{l}\tilde{N} \\
\text { s. } \\
\text { w }\end{array}$ & $\underset{\substack{0 \\
i n}}{\stackrel{0}{N}}$ & $\underset{\text { i }}{\tilde{N}}$ & 总 & 平 & 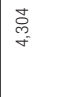 & 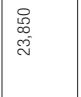 & 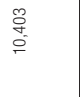 & $\frac{g}{d v}$ & 是 & 号 & $\begin{array}{l}\text { 棠 } \\
\text { b }\end{array}$ & 兑 \\
\hline \multirow{4}{*}{ 帘 } & 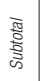 & $\begin{array}{l}\infty \\
\stackrel{\infty}{0} \\
\infty\end{array}$ & \begin{tabular}{l}
$\mathscr{0}$ \\
\hdashline \\
\hdashline
\end{tabular} & $\underset{j}{\stackrel{f}{f}}$ & 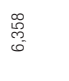 & $\underset{r}{\stackrel{g}{r}}$ & 总 & 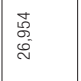 & 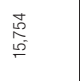 & 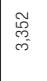 & $\begin{array}{l}\stackrel{8}{\circ} \\
\stackrel{8}{\circ} \\
0 \\
0\end{array}$ & 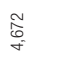 & \begin{tabular}{|l|}
0 \\
$\frac{0}{6}$ \\
\end{tabular} & $\begin{array}{l}\frac{L}{N} \\
\text { s. } \\
\delta\end{array}$ \\
\hline & \begin{tabular}{l}
0 \\
\multirow{2}{0}{}
\end{tabular} & $\stackrel{n}{R}$ & 字 & $\stackrel{?}{\stackrel{2}{r}}$ & ని & F & $\stackrel{\mathscr{\infty}}{\sim}$ & $\underset{\infty}{\infty}$ & $\stackrel{\widetilde{N}}{\stackrel{N}{=}}$ & ڤั & 8 & 志 & 足 & 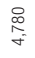 \\
\hline & 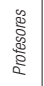 & & & $\stackrel{\sim}{\sim}$ & 总 & $\approx$ & $\bar{\infty}$ & $\underset{\infty}{\infty}$ & $\underset{\substack{\infty \\
=}}{\Sigma}$ & $\frac{7}{6}$ & 电 & 8 & 号 & 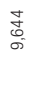 \\
\hline & 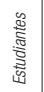 & 总 & 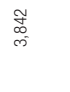 & ০. & 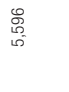 & 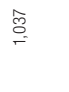 & 志 & \begin{tabular}{|l}
$\infty$ \\
$\stackrel{\infty}{a}$ \\
\multirow{d}{*}{}
\end{tabular} & $\underset{\substack{\mathbb{N} \\
\sim}}{\tilde{N}}$ & 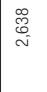 & $\underset{\substack{\mathbb{J} \\
\infty}}{\mathbb{J}}$ & 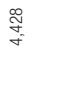 & $\mid \begin{array}{l}\infty \\
0 \\
0 \\
i \infty\end{array}$ & 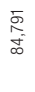 \\
\hline \multirow{4}{*}{ 高 } & $\begin{array}{l}\text { 售 } \\
\text { 害 }\end{array}$ & $\begin{array}{l}: \\
\varpi_{\infty}\end{array}$ & $\begin{array}{l}\frac{m}{\infty} \\
\stackrel{\infty}{*}\end{array}$ & $\begin{array}{l}\infty \\
\infty \\
\infty \\
\infty\end{array}$ & 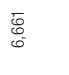 & 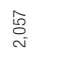 & $\stackrel{\circ}{\stackrel{\circ}{\circ}}$ & 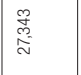 & $\frac{\widetilde{\sigma}}{\square}$ & $\frac{\rho}{\frac{\rho}{\gamma}}$ & $\begin{array}{l}\text { o } \\
\text { S. } \\
0\end{array}$ & 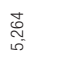 & $\begin{array}{l}\underset{2}{\Omega} \\
\stackrel{N}{N}\end{array}$ & $\begin{array}{l}\frac{0}{0} \\
\stackrel{0}{0} \\
\stackrel{0}{0}\end{array}$ \\
\hline & 产 & 앙 & $\stackrel{\circ}{\stackrel{\circ}{~}}$ & $\stackrel{\infty}{\circ}$ & s & 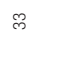 & 另 & $\stackrel{\leftrightarrow}{\circ}$ & 品 & 电 & : & $\infty$ & $\infty$ & \\
\hline & 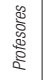 & $\prod_{\infty}^{\infty}$ & $\underset{\sim}{\sim}$ & $\stackrel{8}{\circ}$ & $\Phi$ & 8 & $\stackrel{\mathscr{P}}{\rightleftharpoons}$ & $\begin{array}{l}\text { 寽 } \\
0\end{array}$ & $\stackrel{\sim}{\check{\sigma}}$ & $\stackrel{Z}{N}$ & 兽 & in & $\stackrel{ \pm}{\sim}$ & 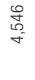 \\
\hline & $\begin{array}{l}\text { 胥 } \\
\text { 密 } \\
\text { 总 }\end{array}$ & $\underset{\infty}{\substack{f \\
\infty}}$ & $\begin{array}{l}\stackrel{\mathscr{J}}{\mathrm{J}} \\
\underset{\forall}{*}\end{array}$ & $\begin{array}{l}\stackrel{8}{\circ} \\
\text { 品 }\end{array}$ & 胥 & $\stackrel{\leftrightarrow}{\stackrel{\leftrightarrow}{\circ}}$ & 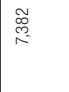 & 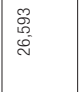 & 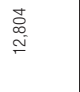 & \begin{tabular}{|l}
$\mathscr{8}$ \\
$\infty$ \\
$\infty$
\end{tabular} & \begin{tabular}{|l}
$\stackrel{2}{2}$ \\
S
\end{tabular} & 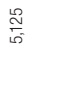 & $\mid \begin{array}{l}\tilde{y} \\
0 \\
\sim\end{array}$ & $\begin{array}{l}\widehat{c} \\
\text { c. } \\
\text { s. }\end{array}$ \\
\hline \multirow{4}{*}{ ڤ్ } & $\begin{array}{l}\text { 敔 } \\
\text { के }\end{array}$ & 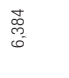 & $\underset{\substack{\mathfrak{j} \\
\multirow{2}{*}{}}}{ }$ & \begin{tabular}{l} 
\& \\
\multirow{+}{*}{}
\end{tabular} & $\begin{array}{c}\stackrel{8}{8} \\
\stackrel{S}{\circ}\end{array}$ & 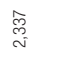 & $\begin{array}{l}0 \\
8 \\
o \\
0 \\
0\end{array}$ & 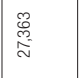 & $\begin{array}{l}\hat{f} \\
\text { of } \\
c\end{array}$ & \begin{tabular}{|l}
$\overline{5}$ \\
0.0 \\
00
\end{tabular} & $\begin{array}{l}\text { 总 } \\
\stackrel{\leftrightarrow}{\sim}\end{array}$ & 胥 & $\frac{d}{\sigma}$ & 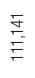 \\
\hline & $\begin{array}{l}\text { s. } \\
0\end{array}$ & 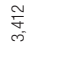 & F & 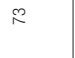 & $\stackrel{\infty}{\infty}$ & N & $=$ & 8 & $\infty$ & $\approx$ & $\stackrel{\Xi}{\rightleftarrows}$ & 욤 & $\bar{\lambda}$ & $\frac{\mathscr{m}}{q}$ \\
\hline & 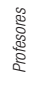 & $\hat{E}$ & 兽 & $\stackrel{\circ}{\sim}$ & $\stackrel{\substack{0 \\
=}}{=}$ & $\cong$ & 㤐 & 志 & 曲 & $\underset{ఝ}{0}$ & 总 & $\stackrel{+}{\circ}$ & 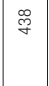 & 惫 \\
\hline & $\begin{array}{l}\text { 胥 } \\
\text { 密 } \\
\text { 悹 }\end{array}$ & 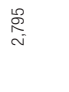 & 曽 & 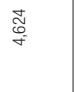 & 品 & $\stackrel{\infty}{\stackrel{\infty}{\sim}}$ & $\begin{array}{l}\sqrt{e} \\
0 \\
0\end{array}$ & \begin{tabular}{|l} 
是 \\
心
\end{tabular} & 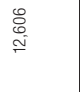 & $\begin{array}{l}\text { 苦 } \\
\text { in }\end{array}$ & $\begin{array}{c}\mathbb{d} \\
\infty \\
\infty \\
\infty\end{array}$ & 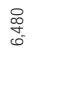 & 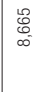 & 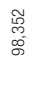 \\
\hline 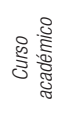 & 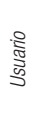 & 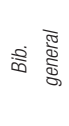 & 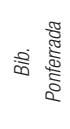 & 离 & $\stackrel{\frac{9}{5}}{\frac{5}{0}}$ & 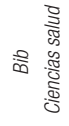 & ஃ & 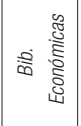 & 这 & 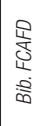 & 言蜜 & 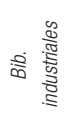 & 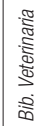 & 迎 \\
\hline \multicolumn{15}{|c|}{$2 z !|| e 007$} \\
\hline
\end{tabular}




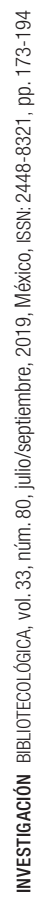

\begin{tabular}{|c|c|c|c|c|c|c|c|c|c|c|c|c|c|}
\hline $\begin{array}{l}\text { 覀 } \\
\text { s. }\end{array}$ & $\begin{array}{l}\infty \\
\stackrel{\infty}{N} \\
\sim\end{array}$ & 总 & $\begin{array}{l}\mathscr{\infty} \\
\stackrel{\infty}{\sim}\end{array}$ & 哭 & $\stackrel{\infty}{\mathscr{D}}$ & $\begin{array}{l}\stackrel{\infty}{\infty} \\
\infty \\
\omega \\
\omega\end{array}$ & 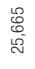 & $\begin{array}{l}\text { 总 } \\
\stackrel{\text { ले }}{=}\end{array}$ & $\begin{array}{l}\infty \\
\stackrel{\infty}{\circ} \\
i\end{array}$ & 号 & 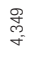 & 跡 & 志 \\
\hline s. & $\frac{\bar{\sigma}}{i 0^{\circ}}$ & 夺 & $\stackrel{\mathscr{\infty}}{\stackrel{\mathscr{D}}{\rightleftharpoons}}$ & 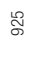 & $\mathscr{\gamma}$ & $\underset{\sim}{\stackrel{S}{S}}$ & 怘 & 喿 & 范 & 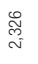 & 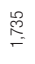 & 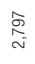 & 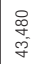 \\
\hline 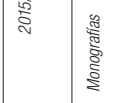 & \begin{tabular}{|l|}
$\infty$ \\
$\stackrel{\infty}{0}$ \\
$\stackrel{0}{0}$
\end{tabular} & 产 & g & $\mathscr{ల}_{\sim}$ & : & 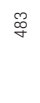 & 苦 & 琶 & 㧝 & 噪 & ळ్ల & 足 & 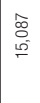 \\
\hline 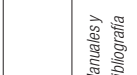 & - & 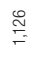 & 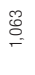 & $\begin{array}{l}\stackrel{8}{8} \\
\dot{f}\end{array}$ & 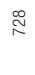 & 亭 & 品 & 总 & 总 & 总 & 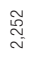 & $\underset{\text { A }}{\text { N }}$ & 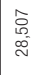 \\
\hline
\end{tabular}

\begin{tabular}{|c|c|c|c|c|}
\hline $\begin{array}{l}\infty \\
\stackrel{\infty}{0} \\
\infty\end{array}$ & : & & & \\
\hline
\end{tabular}

紊

\begin{tabular}{|c|c|c|c|c|c|c|c|c|c|c|c|c|c|}
\hline 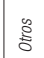 & 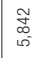 & 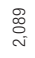 & 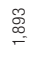 & $\underset{\mathscr{O}}{-}$ & I & 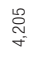 & 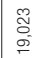 & $\underset{\infty}{\infty}$ & §్లి & 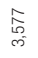 & $\begin{array}{l}: \\
:\end{array}$ & 总 & $\begin{array}{l}\bar{y} \\
\bar{y}\end{array}$ \\
\hline 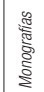 & 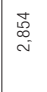 & $\underset{N}{N}$ & t5 & $\frac{8}{d}$ & is & 。 & $\underset{\mid g}{9}$ & & $\bar{y}$ & 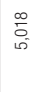 & of & $\bar{\Phi}$ & 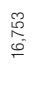 \\
\hline
\end{tabular}

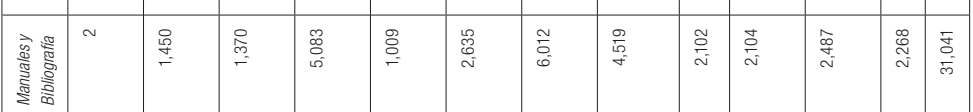

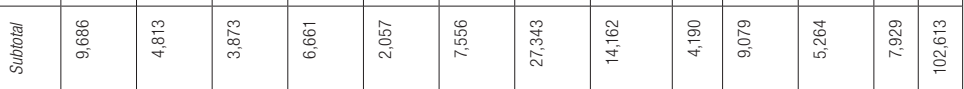

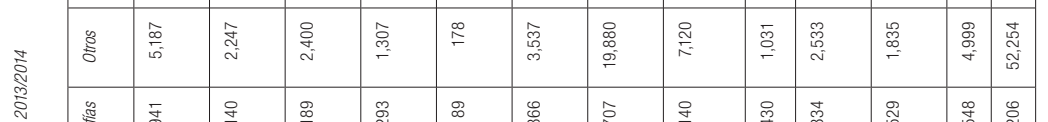

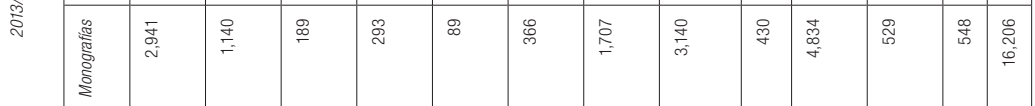

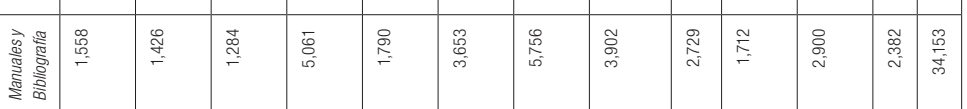

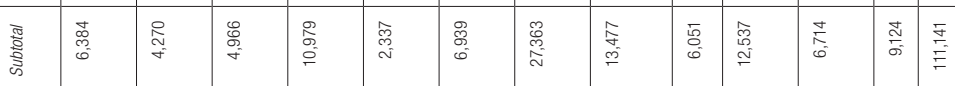

\begin{tabular}{|c|c|c|c|c|c|c|c|c|c|c|c|}
\hline s. & $\frac{\bar{m}}{m}$ & 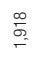 & 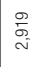 & 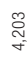 & 疋 & o & $\begin{array}{l}\bar{E} \\
\text { aे }\end{array}$ & a & & & \\
\hline 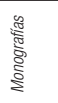 & $\underset{⿱}{g}$ & 命 & ळ & 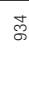 & 욤 & ঃ & 梠 & 0 & 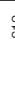 & & \\
\hline 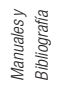 & 음 & 紂 & 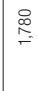 & $\begin{array}{l}\infty \\
\infty \\
\infty\end{array}$ & 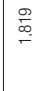 & $\stackrel{\frac{g}{D}}{\frac{\mathrm{m}}{m}}$ & ת) & ల & 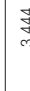 & & \\
\hline
\end{tabular}

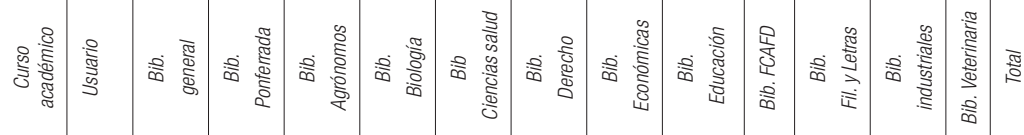


Como se observa en el Cuadro 7, del volumen de transacciones y préstamos en todas las bibliotecas son responsables los estudiantes. No obstante, en algunos cursos se observa, asimismo, un elevado número de préstamos por parte del profesorado. En general, este uso es superior en el caso de las Facultades de Ciencias Sociales y Humanidades, en concreto en las Facultades de Ciencias Económicas, Educación y Filosofía y Letras. Como se observa en el Cuadro 2 y ya pusimos de relieve, el área de Ciencias Sociales de la ULe es fuerte en alumnado.

En el Cuadro 8 se observa un elevado número de préstamos de manuales en todos los centros, acorde con las preferencias ya puestas de relieve de los estudiantes. Los manuales tradicionalmente han sido de uso prioritario para el estudio en las asignaturas científicas y técnicas, de ahí el uso que se observa en los centros donde se imparten estos títulos. En la Gráfica 3 observamos que las cifras más elevadas de préstamos de manuales corresponden a la Facultad de Económicas y Facultad de Biología.

En el Cuadro 8 y en la Gráfica 4 observamos que las monografías son prioritarias en las Facultades de Filosofía y Letras y Educación. La primera concentra las titulaciones del área de Humanidades. Asimismo, es un material de préstamo relevante en la Biblioteca Central que reúne colecciones de monografías de todas las áreas de estudio de la ULe.

El resultado de la última encuesta Ithaka de Estados Unidos (Wolff et al., 2016), entre otros estudios, constata que los humanistas siguen otorgando un papel prioritario a la monografía impresa en sus actividades docentes e investigadoras. Esta preferencia también es común en los ámbitos de las Ciencias Sociales.

Estudios anteriores de los autores (Rodríguez-Bravo et al., 2015a; Rodríguez-Bravo et al., 2015b) constataron que manuales y monografías son considerados documentos esenciales para la docencia en la ULe, mientras que las revistas electrónicas, principalmente en los ámbitos científicos, se consideran imprescindibles para la investigación. En este sentido, además de la tradicional confianza de los científicos en las revistas, cumple un papel trascendental el hecho de que los Big Deals contratados dispongan de contenidos esencialmente de las áreas de Ciencias Puras, de la Naturaleza y de la Salud. 


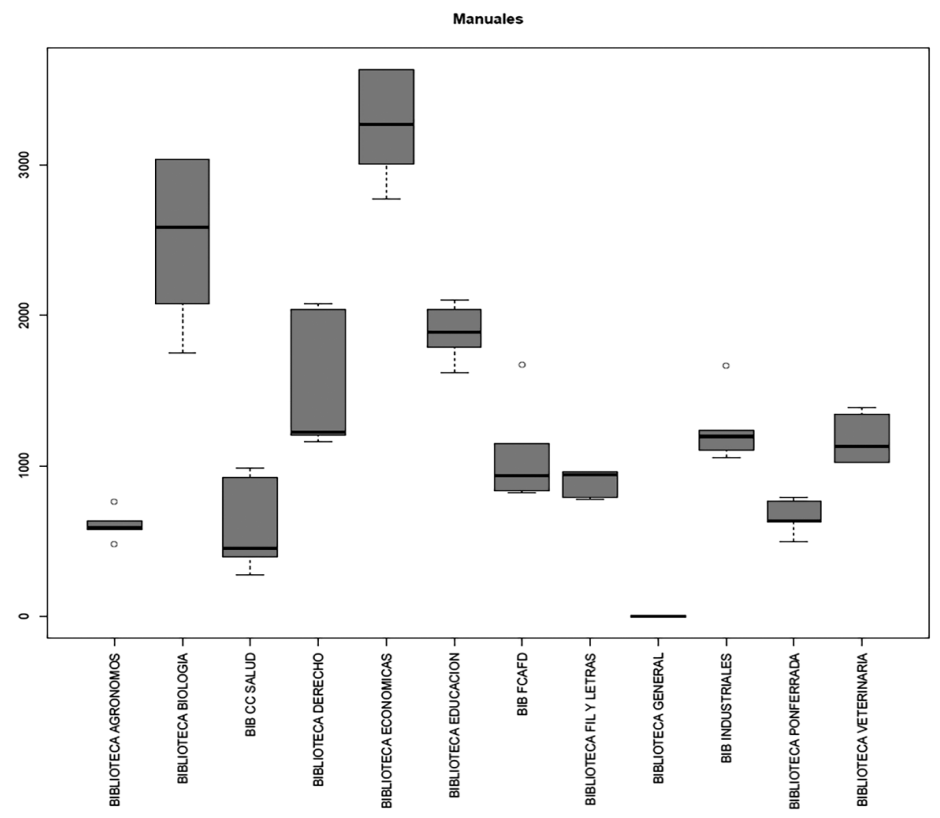

Gráfica 3. Caja del préstamo de manuales y bibliografía recomendada por centros.

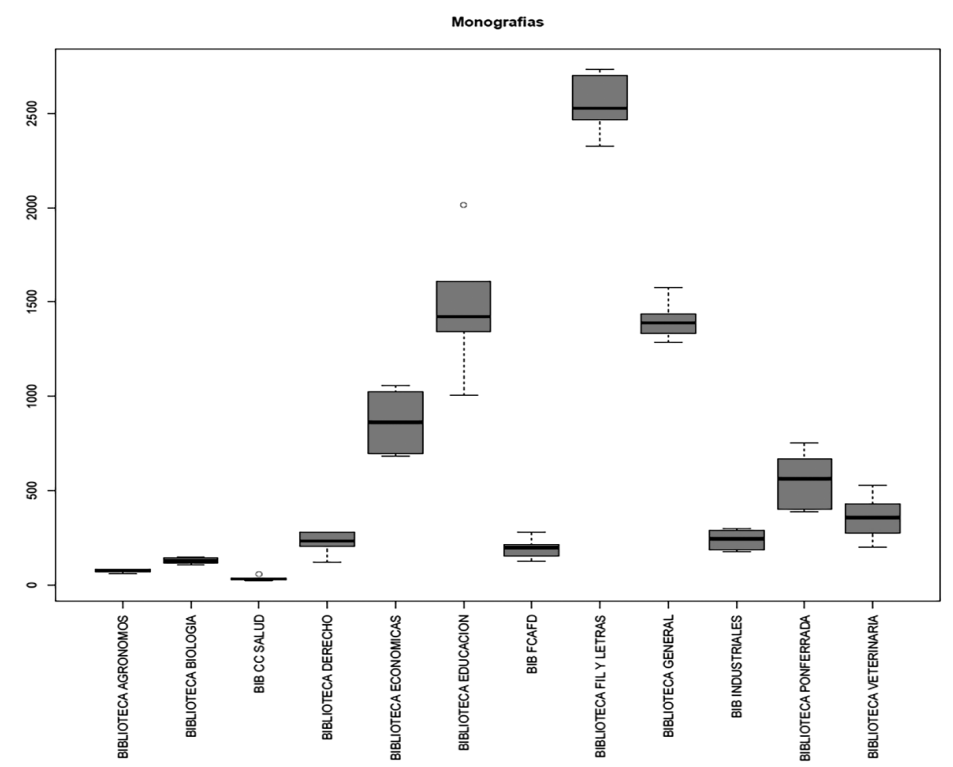




\section{DisCUSIÓN Y CONCLUSIONES}

Si ponemos en relación el total de la colección, según datos de 2012 y 2015, que son los únicos años con datos completos, como se observa en el Cuadro 3, con los usuarios potenciales de los cursos 2012-2013 y 2015-2016 obtenemos una disponibilidad de 37.14 documentos por usuario en 2012-2013 y de 36.22 en 2015-2016.

Durante los últimos años, la colección se ha incrementado de manera muy limitada, debido al descenso del presupuesto de la ULe. No obstante, también se ha producido una ligera disminución de la comunidad universitaria, lo que ha permitido unas ratios bastante sostenidas, aunque se aprecia una ligera disminución.

Por lo que se refiere al movimiento de la colección, en el informe de Fesabid coordinado por Gomez Yañez (2014), se afirmaba que el número de préstamos por usuario en las bibliotecas de instituciones de enseñanza superior fue de 9.7 en 2010. Cifra superior a la hallada en nuestro estudio, que oscila entre 8.15 y 6.96. Es el personal docente e investigador quien más documentos en préstamo utiliza.

El préstamo, al igual que la colección, está dominado por los libros que siguen constituyendo la base esencial de la bibliografía básica recomendada a los estudiantes en las asignaturas. Tanto manuales como monografías, tienen un uso importante en la docencia y en la investigación en las áreas de humanidades y ciencias sociales.

Es cierto que las bibliotecas universitarias han sido conscientes durante décadas de que las monografías impresas no circulan en grandes volúmenes y de que un porcentaje significativo de las monografías no circula en absoluto. Esta situación ya fue puesta de manifiesto en el clásico estudio de Kent et al. (1979) y su bien conocida regla del $80 / 20$, que indica que el 80 por ciento de los préstamos de la biblioteca se realiza con el 20 por ciento de la colección. Un informe más reciente de OCLC (Gammon y O’Neill, 2011) sobre la colección del consorcio OhioLink, señalaba que la ratio está todavía más descompensada, con el 80 por ciento del movimiento realizado sobre el 6 por ciento de la colección.

Son numerosos los estudios que analizan la circulación de monografías y que muestran que los porcentajes de monografías que circulan no superan el 50 por ciento. Es el caso de los realizados en el entorno estadounidense por Cornell University (2010) y por Cramer (2013). También son importantes los trabajos que inciden en que la circulación de monografías está disminuyendo. Entre otros, podemos señalar los de Kyrillidou y Morris (2011) y Rose-Wiles (2013). 
La carencia de cifras elevadas de circulación, como señala Proctor (2015), es la razón por la que los programas de adquisición a demanda del usuario (PDA) constituyen un modelo deseable, ya que implican que al menos cada ítem de la colección circulará como mínimo una vez. Además, algunos estudios demuestran que los títulos adquiridos a través del modelo PDA tienden a circular más que los elegidos, siguiendo métodos de selección tradicionales. En este mismo sentido, el análisis hecho por Cheung et al. (2011) concluye que los libros que son sacados en préstamo nada más comprados continuarán circulando en el futuro.

La adquisición adaptada a la demanda de los usuarios es una opción atractiva para los bibliotecarios que necesitan ahorrar costes, gastar menos en libros no utilizados, disponer de más espacio y desarrollar una colección que realmente responda a las necesidades de los usuarios.

La ULe ha evolucionado hacia este modelo durante la última década. Se adquieren los documentos que los profesores solicitan para su uso y el de sus alumnos, y las adquisiciones se complementan con las decisiones de compra de los bibliotecarios, fundadas principalmente en la demanda de un mayor número de ejemplares de manuales para uso de los estudiantes.

Ante la necesidad de demostrar la responsabilidad en la inversión de sus presupuestos y de rendir cuentas ante los administrados, la política del "just in time" ha sustituido a la del "just in case". Si las bibliotecas y los gestores de las colecciones desean competir con otros servicios focalizados en el usuario, necesitan integrar a estos en la construcción de la colección que anteriormente era responsabilidad exclusiva del personal bibliotecario (Chadwell, 2009).

\section{REFERENCIAS}

Chadwell, F. A. (2009) "What's Next for Collection Management and Managers? User-Centered”. Collection Management, 34: 69-78.

Cheung, S., T. Chung y F. Nesta (2011). "Monograph circulation over 15-year period in a liberal arts university". Library Management, 32 (6): 419-434.

Cornell University Library (2010). Report of the Collection Development Executive Committee Task Force on Print Collection Usage. Ítaca: Cornell University Library, <http://staffweb.library.cornell.edu/system/files/CollectionUsageTF_ReportFinal11-22-10.pdf>.

Cramer, J. C. (2013). “All about demand-driven acquisition”, Serials Librarian, 65 (1): 87-97.

Junta de Castilla y León (jcyl) (2018). "Estadística universitaria de Castilla y León". Educacyl. Portal de Educación. <http://www.educa.jcyl.es/universidad/es/estadistica-universitaria-castilla-leon $>$. 
Gammon, J. y E.T. O’Neill (2011). Ohio Link Collection \& Circulation Analysis Project 2011, <http://www.oclc.org/content/dam/research/publications/library/2011/2011-06.pdf? urlm=162957>.

Gomez Yañez, J. A. (coord.) (2014). The economic and social value of information services: libraries. Madrid: Fesabid.

Kent, A., J. Cohen, K. L. Montgomery, K. G. Williams, S. Bulick, R. R. Flynn, W. N. Sabor y U. Mansfield (1979). Use of Library Materials. Nueva York: Marcel Dekker.

Kyrillidou, M y S. Morris (2011). ARL statistics 2008-2009. Washington, D.C.: Association of Research Libraries.

Martell, C. (2008). "The Absent User: Physical Use of Academic Library Collections and Services Continues to Decline 1995-2006". The Journal of Academic Librarianship, 34 (5): 400-407. <http://www.sciencedirect.com/science/article/pii/ S0099133308001018>.

Proctor, J. (2015). "Demand-driven acquisition and the sunk cost model". Collection Building, 34 (1): 2-5.

Red de Bibliotecas Universitarias Española (Rebiun) (2018). <http://www.rebiun.org/>.

Rodríguez-Bravo, B. y F. Rodríguez-Sedano (2016). "Trends in Library Collection Circulation in Spanish Universities. The case of the University of Leon”. Library Resources and Technical Services, 60 (4): 248-258.

Rodríguez-Bravo, B., A.R. Pacios, M. Vianello-Osti, M. Moro-Cabero y M. de la Mano-González (2015a). "Digital Transition of Teaching-Learning Resources at Spanish Universities". El Profesional de la Información (noviembre-diciembre), 24 (6): $733-744$.

Rodríguez-Bravo, B., Mª L. Alvite-Díez y I. Olea. (2015b). “La utilización de las revistas electrónicas en la Universidad de León (España): hábitos de consumo y satisfacción de los investigadores". Investigación Bibliotecológica: archivonomía, bibliotecología e información, 29 (66): 17-55.

Rose-Wiles, L. (2011). "The high cost of science journals: A case study and discussion”. Journal of Electronic Resources Librarianship, 23: 219-241.

Rose-Wiles, L. (2013). "Are print books dead? An investigation of book circulation at a mid-sized academic library”. Technical Services Quarterly, 30 (2): 129-152.

Simón-Martín, J. A. Arias-Coello y C. Simón-Blas (2016). "The impact of the economic crisis on Spanish university libraries". Revista Española de Documentación Cientifica, 39 (3), e142.

Wolff, C., A. B. Rod, R. C. Schonfeld (2016). Ithaka S+R US Faculty survey 2015. Nueva York: Ithaka S+R, <https://doi.org/10. 18665/sr.277685>. 


\section{Datos globales de préstamos totales por tipo de material y por usuario}

Curso académico 2011/2012

\begin{tabular}{|c|c|c|c|c|c|c|c|c|c|}
\hline \multirow{11}{*}{ 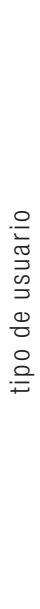 } & Tipo de material & $\begin{array}{c}\text { Manualesy } \\
\text { bibliografía } \\
\text { rec. }\end{array}$ & Monog. & $\begin{array}{c}\text { Material } \\
\text { especial } \\
\text { (cd, audio,-- } \\
\text { video) }\end{array}$ & $\begin{array}{c}\text { Tesis y } \\
\text { trabajos fin } \\
\text { de grado }\end{array}$ & Referenc. & $\begin{array}{l}\text { Material } \\
\text { cartográf. }\end{array}$ & $\begin{array}{c}\text { Pub. } \\
\text { periódicas }\end{array}$ & $\begin{array}{c}\text { Colecciones } \\
\text { especiales }\end{array}$ \\
\hline & Estudiantes & 32,340 & 11,090 & 185 & 4,977 & 479 & 78 & 2,198 & 2 \\
\hline & Biblioteca & 139 & 187 & 12 & 20 & 0 & 1 & 35 & 1 \\
\hline & Sala de consulta & 0 & 8 & 0 & 68 & 0 & 0 & 2 & 0 \\
\hline & Millennium & 0 & 8 & 68 & 9 & 0 & 0 & 0 & 0 \\
\hline & $\begin{array}{l}\text { Personal Admin./ } \\
\text { serv. }\end{array}$ & 359 & 630 & 56 & 46 & 21 & 0 & 95 & 0 \\
\hline & Profesores & 2,116 & 3,356 & 89 & 47 & 126 & 0 & 965 & 25 \\
\hline & $\begin{array}{l}\text { Préstamo } \\
\text { interbibliotec. }\end{array}$ & 11 & 162 & 2 & 8 & 0 & 3 & 35 & 2 \\
\hline & Usuarios externos & 209 & 380 & 44 & 15 & 0 & 0 & 71 & 0 \\
\hline & $\begin{array}{l}\text { Usuarios } \\
\text { temporales }\end{array}$ & 11 & 59 & 1 & 0 & 1 & 0 & 0 & 0 \\
\hline & Total & 35,185 & 15,880 & 398 & 5,181 & 627 & 82 & 3,401 & 30 \\
\hline
\end{tabular}

\section{Curso académico 2012/2013}

\begin{tabular}{|c|c|c|c|c|c|c|c|c|c|}
\hline \multirow{11}{*}{ 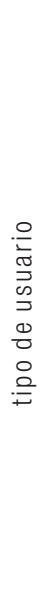 } & Tipo de material & $\begin{array}{c}\text { Manuales y } \\
\text { bibliografía } \\
\text { rec. }\end{array}$ & Monog. & $\begin{array}{c}\text { Material } \\
\text { especial } \\
\text { (cd, audio,- } \\
\text { video) }\end{array}$ & $\begin{array}{c}\text { Tesisy } \\
\text { trabajos fin } \\
\text { de grado }\end{array}$ & Referenc. & $\begin{array}{c}\text { Material } \\
\text { cartográt. }\end{array}$ & $\begin{array}{c}\text { Pub. } \\
\text { periódicas }\end{array}$ & $\begin{array}{c}\text { Colecciones } \\
\text { especiales }\end{array}$ \\
\hline & Estudiantes & 31,532 & 11,745 & 177 & 4,441 & 412 & 42 & 1,700 & 1 \\
\hline & Biblioteca & 142 & 170 & 7 & 20 & 0 & 1 & 11 & 1 \\
\hline & Sala de consulta & 3 & 8 & 0 & 57 & 0 & 0 & 2 & 0 \\
\hline & Millennium & 0 & 8 & 5 & 0 & 0 & 0 & 0 & 0 \\
\hline & $\begin{array}{l}\text { Personal Admin./ } \\
\text { serv. }\end{array}$ & 382 & 566 & 15 & 21 & 21 & 0 & 77 & 1 \\
\hline & Profesores & 2,485 & 3,072 & 67 & 19 & 152 & 0 & 965 & 22 \\
\hline & $\begin{array}{l}\text { Préstamo } \\
\text { interbibliotec. }\end{array}$ & 11 & 139 & 2 & 5 & 0 & 2 & 29 & 2 \\
\hline & Usuarios externos & 338 & 377 & 40 & 10 & 0 & 0 & 67 & 0 \\
\hline & $\begin{array}{l}\text { Usuarios } \\
\text { temporales }\end{array}$ & 11 & 69 & 1 & 0 & 1 & 0 & 0 & 0 \\
\hline & Total & 34,904 & 16,154 & 314 & 4,573 & 586 & 45 & 2,851 & 27 \\
\hline
\end{tabular}


Curso académico 2013/2014

\begin{tabular}{|c|c|c|c|c|c|c|c|c|c|}
\hline \multirow{11}{*}{ 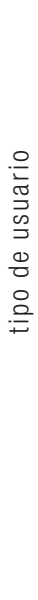 } & Tipo de material & $\begin{array}{c}\text { Manuales y } \\
\text { bibliografía } \\
\text { rec. }\end{array}$ & Monog. & $\begin{array}{c}\text { Material } \\
\text { especial } \\
\text { (cd, audio,- } \\
\text { video) }\end{array}$ & $\begin{array}{c}\text { Tesis y } \\
\text { trabajos fin } \\
\text { de grado }\end{array}$ & Referenc. & $\begin{array}{l}\text { Material } \\
\text { cartográf. }\end{array}$ & $\begin{array}{c}\text { Pub. } \\
\text { periódicas }\end{array}$ & $\begin{array}{c}\text { Colecciones } \\
\text { especiales }\end{array}$ \\
\hline & Estudiantes & 30,064 & 12,026 & 476 & 3,317 & 396 & 30 & 1,606 & 7 \\
\hline & Biblioteca & 104 & 217 & 19 & 30 & 0 & 0 & 62 & 0 \\
\hline & Sala de consulta & 4 & 2 & 0 & 26 & 0 & 0 & 4 & 0 \\
\hline & Millennium & 240 & 268 & 18 & 23 & 9 & 1 & 50 & 0 \\
\hline & $\begin{array}{l}\text { Personal Admin./ } \\
\text { serv. }\end{array}$ & 166 & 164 & 152 & 14 & 6 & 0 & 18 & 0 \\
\hline & Profesores & 2,528 & 3,074 & 32 & 47 & 140 & 0 & 1,043 & 14 \\
\hline & $\begin{array}{l}\text { Préstamo } \\
\text { interbibliotec. }\end{array}$ & 11 & 281 & 7 & 4 & 0 & 0 & 22 & 0 \\
\hline & Usuarios externos & 317 & 532 & 28 & 38 & 3 & 0 & 73 & 0 \\
\hline & $\begin{array}{l}\text { Usuarios } \\
\text { temporales }\end{array}$ & 13 & 9 & 1 & 0 & 0 & 0 & 2 & 0 \\
\hline & Total & 33,447 & 16,573 & 733 & 3,499 & 554 & 31 & 2,880 & 21 \\
\hline
\end{tabular}

Curso académico 2014/2015

\begin{tabular}{|c|c|c|c|c|c|c|c|c|c|}
\hline & Tipo de material & $\begin{array}{c}\text { Manuales y } \\
\text { bibliografía } \\
\text { rec. }\end{array}$ & Monog. & $\begin{array}{c}\text { Material } \\
\text { especial } \\
\text { (cd, audio,- } \\
\text { video) }\end{array}$ & $\begin{array}{c}\text { Tesis y } \\
\text { trabajos fin } \\
\text { de grado }\end{array}$ & Referenc. & $\begin{array}{l}\text { Material } \\
\text { cartográf. }\end{array}$ & $\begin{array}{c}\text { Pub. } \\
\text { periódicas }\end{array}$ & $\begin{array}{c}\text { Colecciones } \\
\text { especiales }\end{array}$ \\
\hline \multirow{10}{*}{ 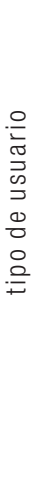 } & Estudiantes & 26,515 & 11,722 & 392 & 2,621 & 188 & 31 & 1,228 & 10 \\
\hline & Biblioteca & 169 & 326 & 8 & 27 & 2 & 0 & 10 & 2 \\
\hline & Sala de consulta & 29 & 7 & 0 & 32 & 0 & 0 & 6 & 0 \\
\hline & Millennium & 261 & 342 & 30 & 8 & 7 & 5 & 46 & 0 \\
\hline & $\begin{array}{l}\text { Personal Admin./ } \\
\text { serv. }\end{array}$ & 185 & 158 & 9 & 4 & 7 & 0 & 20 & 0 \\
\hline & Profesores & 2,767 & 3,184 & 59 & 55 & 163 & 2 & 1,167 & 16 \\
\hline & $\begin{array}{l}\text { Préstamo } \\
\text { interbibliotec. }\end{array}$ & 14 & 335 & 5 & 1 & 1 & 0 & 3 & 0 \\
\hline & Usuarios externos & 674 & 800 & 23 & 12 & 3 & 1 & 85 & 3 \\
\hline & $\begin{array}{l}\text { Usuarios } \\
\text { temporales }\end{array}$ & 36 & 13 & 0 & 0 & 0 & 0 & 13 & 0 \\
\hline & Total & 30,650 & 16,887 & 526 & 2,760 & 371 & 39 & 2,578 & 31 \\
\hline
\end{tabular}


Curso académico 2015/2016

\begin{tabular}{|c|c|c|c|c|c|c|c|c|c|}
\hline \multirow{10}{*}{ 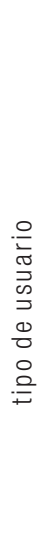 } & Tipo de material & $\begin{array}{c}\text { Manualesy } \\
\text { bibliografía } \\
\text { rec. }\end{array}$ & Monog. & $\begin{array}{c}\text { Material } \\
\text { especial } \\
\text { (cd, audio,- } \\
\text { video) }\end{array}$ & $\begin{array}{c}\text { Tesisy } \\
\text { trabajos fin } \\
\text { de grado }\end{array}$ & Referenc. & $\begin{array}{l}\text { Material } \\
\text { cartográt. }\end{array}$ & $\begin{array}{c}\text { Pub. } \\
\text { periódicas }\end{array}$ & $\begin{array}{c}\text { Colecciones } \\
\text { especiales }\end{array}$ \\
\hline & Estudiantes & 24,543 & 10,493 & 187 & 1,775 & 177 & 19 & 1,107 & 1 \\
\hline & Biblioteca & 185 & 240 & 3 & 30 & 7 & 3 & 51 & 2 \\
\hline & Sala de consulta & 64 & 9 & 0 & 17 & 0 & 0 & 11 & 0 \\
\hline & Millennium & 48 & 59 & 3 & 0 & 2 & 1 & 11 & 0 \\
\hline & $\begin{array}{l}\text { Personal Admin./ } \\
\text { serv. }\end{array}$ & 336 & 415 & 34 & 4 & 37 & 9 & 40 & 5 \\
\hline & Profesores & 2,379 & 2,892 & 65 & 70 & 141 & 8 & 861 & 10 \\
\hline & $\begin{array}{l}\text { Préstamo } \\
\text { interbibliotec. }\end{array}$ & 21 & 286 & 3 & 7 & 1 & 0 & 10 & 0 \\
\hline & Usuarios externos & 800 & 973 & 7 & 7 & 2 & 0 & 63 & 0 \\
\hline & Total & 28,376 & 15,367 & 302 & 1,910 & 367 & 40 & 2,154 & 18 \\
\hline
\end{tabular}

Para citar este texto:

Rodríguez-Bravo, Blanca y Francisco Jesus Rodríguez-Sedano. 2019. "La circulación de libros en la Biblioteca de la Universidad de León (España)". Investigación Bibliotecológica: archivonomía, bibliotecología e información 33 (80): 173-194.

http://dx.doi.org/10.22201/iibi.24488321xe.2019.80.58026 